CASE REPORT

\title{
Unusual cause of infective discitis in an adolescent
}

\author{
M K Sayana, A J Chacko, R C Mc Givney
}

Postgrad Med J 2003;79:237-238

The first case of infective discitis caused by Eikenella corrodens in an adolescent is presented. The need for anaerobic cultures when dealing with infective pathology in the spine is stressed. A 14 year old boy presented with acute exacerbation of back pain, which showed characteristics of infective discitis after magnetic resonance imaging. Computed tomography guided biopsy grew $E$ corrodens in anaerobic cultures that was sensitive to ampicillin, coamoxiclav, cefadroxil, and cefotaxime. This patient responded well to co-amoxiclav and recovered without any surgical intervention.

$E_{\text {o }}$ ikenella corrodens is a slow growing, non-motile, facultative Gram negative bacillus, which is a commensal of the oral cavity, and intestinal and genital tracts. There are case reports of adult spine infections (vertebral osteomyelitis and discitis) ${ }^{12}$ caused by eikenella, but none of spinal infection in children and adolescents. ${ }^{3}$ We report here the first case of discitis secondary to this organism in an adolescent.

The various clinical manifestations caused by this organism include head and neck, pulmonary, intra-abdominal, cutaneous, and skeletal infections, endocarditis, and pelvic abscess. ${ }^{4}$ Rare and interesting presentations like osteomyelitis in a chronic nail biter, ${ }^{5}$ osteomyelitis due to toothpick injury, ${ }^{67}$ and vertebral osteomyelitis due to pharyngeal injury caused by a fish bone ${ }^{2}$ have been reported.

\section{CASE REPORT}

A 14 year old boy had an insidious, atraumatic onset of back pain over a three month period. He had been treated with non-steroidal anti-inflammatories for three weeks. He presented to the accident and emergency department after an acute exacerbation of the back pain. The pain was constant, radiating to the left buttock and thigh, worsened by movements, coughing and sneezing, relieved by rest and lying flat.

Clinical examination revealed scoliosis and paraspinal spasm. There was localised tenderness at the L4/L5 area. Straight leg raising was $20^{\circ}$ on both sides. There was no neurological deficit.

Radiography confirmed the lower lumbar scoliosis and there were bridging osteophytes at L4/L5 on the left suggesting that the scoliosis was chronic (figs 1 and 2). Minor loss of disc space was demonstrated at L4/L5 and L5/S1. No other acute pathology was seen.

Investigations showed a white cell count of $6 \times 10^{9}$ cells/l, erythrocyte sedimentation rate $30 \mathrm{~mm} /$ hour, C-reactive protein 15, rheumatoid factor was negative, and blood cultures were negative.

Magnetic resonance imaging (MRI) was done in two days and showed loss of height in the lumbar $4 / 5$ disc associated with high signal on $\mathrm{T} 2 /$ short $\mathrm{Tl}$ inversion recovery (STIR) images and low signal on Tl images. There was associated loss of cortical margins of the adjacent vertebrae, particularly on the left side, with high signal on T2/STIR images and low sig-

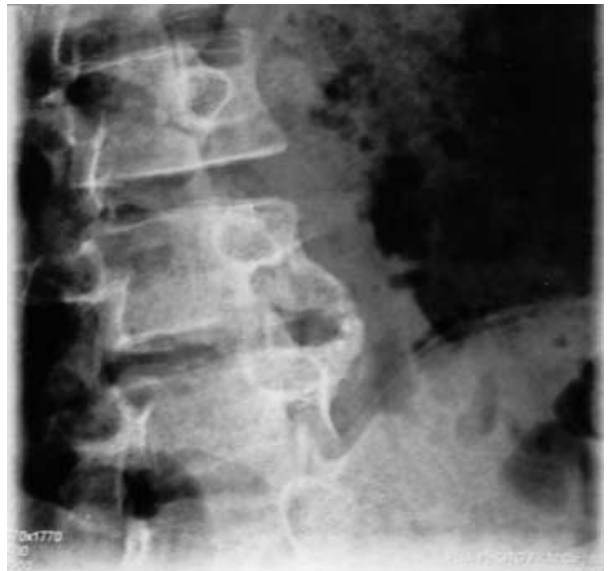

Figure 1 Oblique view of lumbar spine.

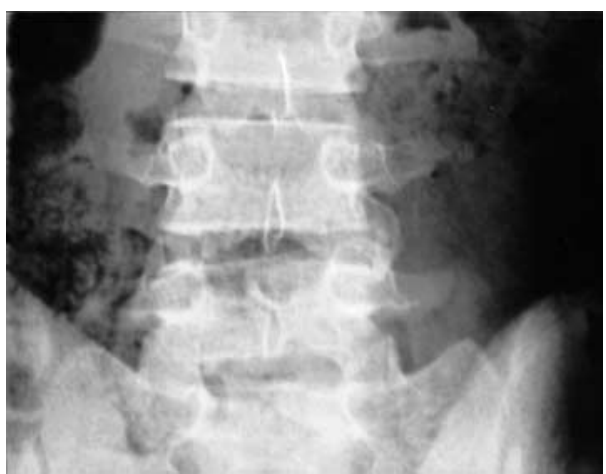

Figure 2 Radiograph (anteroposterior view).

nal on Tl images in the bone marrow consistent with oedema (figs 3 and 4). High signal extended into and narrowed the exit foramina on the left, touching the exiting nerve root. Appearances were consistent with infective discitis and nerve root compression.

Computed tomography guided biopsy produced fluid from the disc, which appeared to be blood stained pus and was sent for microbiology. Histology showed fragmented bony bits and had no diagnostic features. The patient was then started on intravenous cefuroxime $750 \mathrm{mg}$ three times a day.

Microbiology provisionally showed Gram negative rods, sensitive to ampicillin, co-amoxiclav, cefadroxil, and cefotaxime. The patient was started on oral co-amoxiclav (as Augmentin) and responded well to the antibiotic treatment, which was continued for eight weeks. He improved symptomatically and was symptom-free after eight weeks; he has been followed up for six months.

The growths were sent to a reference laboratory for species identification and were reported as Eikenella corrodens (about 10 days after the biopsy). 


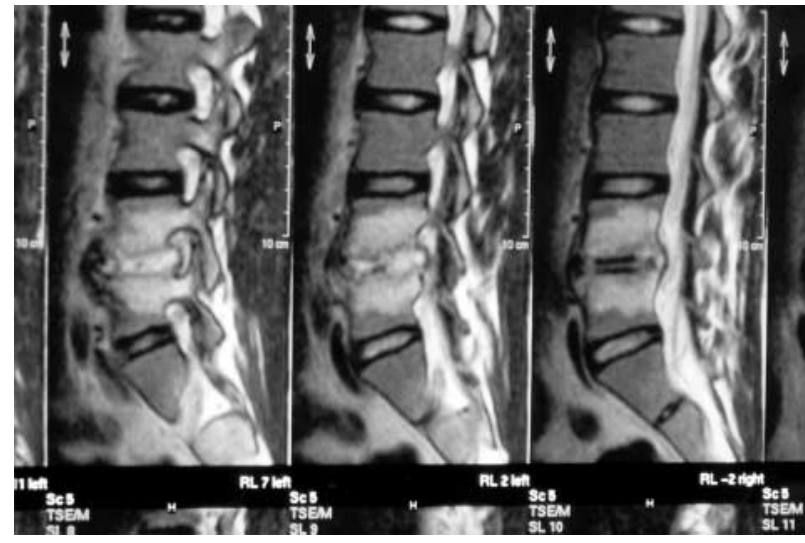

Figure 3 MRI scan: sagittal T2-weighted view.

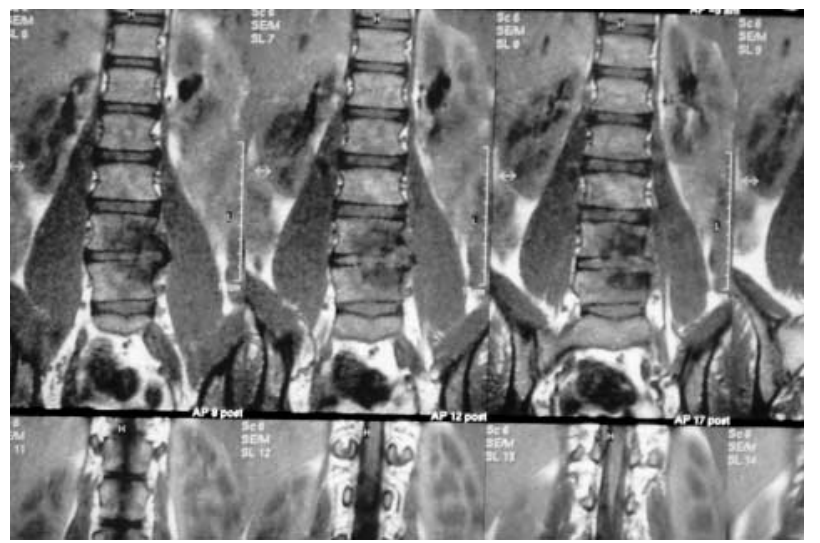

Figure 4 MRI scan: coronal view.

\section{DISCUSSION}

Infective discitis in healthy individuals is a rare occurrence; in children it is commonly seen between 6 months and 3 years.
The most common organism causing discitis is Staphylococcus aureus, followed by Escherichia coli, pseudomonas, streptococci, and klebsiella. This patient was otherwise healthy and had no other medical or dental conditions. Retrospective history did not reveal any human bite injuries or dental extractions. Paul and Patel, in their review of 54 cases of infection caused by eikenella in children and adolescents, did not report discitis in any of the patients. ${ }^{3}$ We stress the importance of anaerobic cultures and in this case the cultures from the computed tomography guided biopsy grew these organisms. E corrodens is best grown on blood agar where it forms small greyish colonies in $5 \%-10 \%$ carbon dioxide. This patient responded well to the antibiotics and did not require any surgical intervention.

\section{Authors' affiliations}

M K Sayana, A J Chacko, R C Mc Givney, Department of

Orthopaedics, Fairfield General Hospital, Rochdale Old Road, Bury,

Lancashire BL9 7TA, UK

Correspondence to: Dr Sayana; sayanamk@hotmail.com

Submitted 9 August 2002

Accepted 30 October 2002

\section{REFERENCES}

1 Lehman CR, Decky JE, Hu SS. Eikenella corrodens. Vertebral osteomyelitis secondary to direct inoculation: case report. Spine 2000:25: $1185-7$.

2 Noordeen MH, Godfrey LW. Case of an unusual cause of low back pain. Intervertebral discitis caused by Eikenella corrodens. Clin Orthop 1992;280: 175-8

3 Paul K, Patel SS. Eikenella corrodens infections in children and adolescents: case reports and review of the literature. Clin Infect Dis 2001;33:54-61.

4 Sheng WS, Hsueh PR, Hung CC, et al. Clinical features of patients with invasive Eikenella corrodens infections and microbiological characteristics of the causative isolates. Eur J Clin Microbiol Infect Dis 2001;20:231-6.

5 Sagerman SD, Lourie GM. Eikenella osteomyelitis in a chronic nail biter: a case report. J Hand Surg [Am] 1995;20:71-2.

6 Seigel IM Identification of non metallic foreign bodies in soft tissue: Eikenella corrodens metatarsal osteomyelitis due to a retained toothpick. A case report. J Bone Joint Surg Am 1992;74:1408-10.

7 Robinson LG, Kurtis AP. Tale of a toothpick: Eikenella corrodens osteomyelitis. Infection 2000;28:332-3. 